\title{
EXCITON STATES IN TYPE-II ZnSe/BeTe QUANTUM WELLS
}

\author{
A.V. Platonov ${ }^{a}$, D.R. YAKovLeV ${ }^{a, b}$, U. ZehNDER ${ }^{b}$, V.P. KochereshKo ${ }^{a}$,

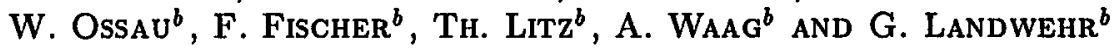 \\ ${ }^{a}$ A.F. Ioffe Physico-Technical Institute, Russian Academy of Sciences \\ St. Petersburg, Russia \\ ${ }^{b}$ Physikalisches Institut der Universität Würzburg, 97074 Würzburg, Germany
}

We present an optical investigation of novel heterostructures based on beryllium chalcogenides with a type-I and type-II band alignment. In the type-II quantum well structures ( $\mathrm{ZnSe} / \mathrm{BeTe}$ ) we observed a strong exciton transition involving an electron confined in the conduction band well and a hole localized in the valence band barrier (both in $\mathrm{ZnSe}$ layer). This transition is drastically broadened by the temperature increase due to enhanced exciton-acoustic phonon interaction.

PACS numbers: 78.66.Hf, 78.20.Ls

Semiconductor heterostructures based on beryllium chalcogenides are relatively new and very promising materials for applications and fundamental studies. Recently these compounds were successfully used for creating light emitting diodes for the blue spectral range [1]. Also heterostructures of high quality with a positive and negative valence band offset are grown on the base of beryllium chalcogenides. These structures can considerably increase faculties for band-structure engineering.

The structures studied were grown by molecular-beam epitaxy on GaAs substrates with (100) orientation. Two types of samples were fabricated: (i) sample 1: 70- $\AA$-thick $\mathrm{ZnSe} / \mathrm{ZnBeSe} \mathrm{QW}$, which has a type-I band alignment, i.e., electron and hole have a minimal energy in ZnSe layer; (ii) sample 2: $160-\AA$-thick $\mathrm{ZnSe} / \mathrm{BeTe}$ quantum well $(\mathrm{QW})$. This system has a type-II band alignment with a very large confinement potential for electron (about $2.0 \mathrm{eV}$ ), which is localized in $\mathrm{ZnSe}$, and for hole $(0.9 \mathrm{eV})$, with minimum of its energy in BeTe layers.

A band diagram of the type-II QW structure $\mathrm{ZnSe} / \mathrm{BeTe}$ is shown in Fig. 1. There are two types of excitonic transitions in such structure: (i) spatially indirect with the energy of about $1.95 \mathrm{eV}$ and (ii) direct transition inside the $\mathrm{ZnSe}$ layer. In the case of large negative valence band offset, the hole can be effectively localized above the barrier because of the strong above-barrier reflection [2]. The energy spectrum of such states is quasi-discrete. In the present paper we study optical properties of the direct exciton states in the type-II QW structures. 


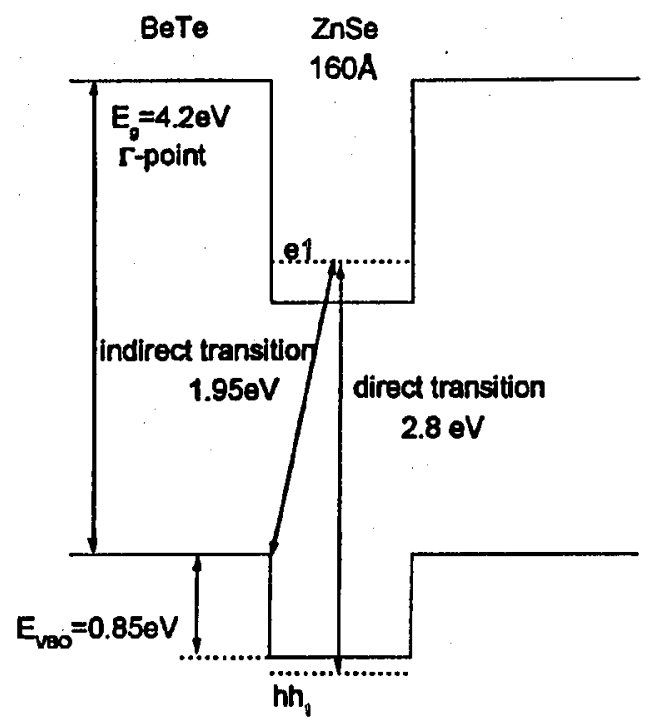

Fig. 1. Band diagram of the type-II QW structure $\mathrm{ZnSe} / \mathrm{BeTe}$.

Reflectivity and photoluminescence spectra (PL) at $1.6 \mathrm{~K}$ are presented in Fig. 2a, b for samples 1 and 2, respectively. The reflectivity spectrum shows three resonances for the type-I QW. These resonances can be identified as heavy-hole and light-hole excitons and a donor bound exciton. At a low temperature the dominating $\mathrm{PL}$ peak $(2.822 \mathrm{eV})$ corresponds to the bound exciton state. A strong resonance feature was observed in the reflectivity spectrum at the energy exceeding the ZnSe band gap for the type-II structures. We identify it as the direct exciton consisting of a confined electron state and a quasi-localized above-barrier hole state in the $\mathrm{ZnSe}$ layer.

Using magneto-optical measurements we have determined an exciton binding energy for both structures: $E_{\mathrm{X}}^{\mathrm{L}}=25 \mathrm{meV}, E_{\mathrm{X}}^{\mathrm{II}}=14 \mathrm{meV}$. This value for the type-II is smaller than for bulk $\mathrm{ZnSe}$, where it is $E_{\mathrm{X}}^{\text {bulk }}=18 \mathrm{meV}$. Temperature dependences of the exciton line width for both type-I and type-II structures are plotted in Fig. 2. A drastic increase in the exciton line width for the type-II QW was observed at low temperatures $(T<100 \mathrm{~K})$ in contrary with that dependence for the type-I QWs. This behavior evidences enhancement of exciton-acoustic phonon interaction in the type-II QWs.

The temperature dependence of the exciton damping (i.e., exciton line width) is generally expressed in the following form:

$$
\Gamma(T)=\Gamma(0)+\gamma_{\mathrm{AC}} T+\gamma_{\mathrm{LO}} n(T),
$$

where $\Gamma(0)$ is the exciton damping at zero temperature, $\gamma_{\mathrm{AC}}$ and $\gamma_{\mathrm{LO}}$ are the constants of the exciton interaction with acoustical and optical phonons, $T$ is a temperature, $n(T)$ is the Planc!!!!k distribution. Such $\Gamma(T)$ dependence for the type-I structure is plotted in Fig. 3 by a dotted line with the parameters: $\gamma_{\mathrm{AC}}=$ $0.018 \mathrm{meV} / \mathrm{K}, \gamma_{\mathrm{LO}}=12 \mathrm{meV}, \Gamma(0)=2.8 \mathrm{meV}$. 


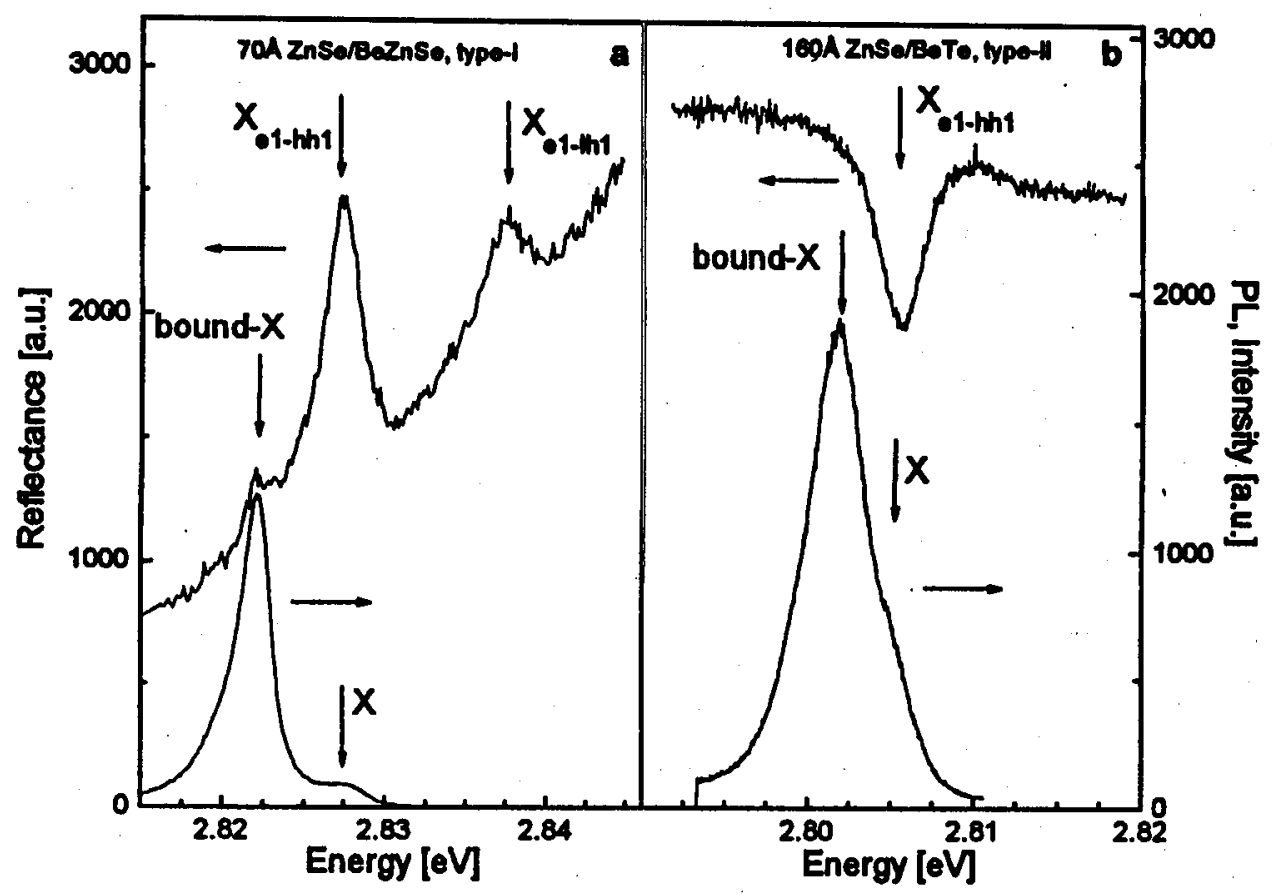

Fig. 2. Photoluminescence and reflectivity spectra measured at $1.6 \mathrm{~K}$ for the type-I (a) and the type-II (b) structures. Excitonic parameters determined from reflectivity spectra [3] are the following: (i) in a $\mathrm{ZnSe} / \mathrm{BeZnSe} \mathrm{QW}$ resonance energy is $\hbar \omega_{0}=2.828 \mathrm{eV}$, oscillator strength $\hbar \omega_{\mathrm{LT}}=1.2 \mathrm{meV}$, damping $\hbar \Gamma=1.3 \mathrm{meV}$; (ii) in a $\mathrm{ZnSe} / \mathrm{BeTe} \mathrm{QW}$ $\hbar \omega_{0}=2.805 \mathrm{eV}, \hbar \omega_{\mathrm{LT}}=1.05 \mathrm{meV}, \hbar \Gamma=1.6 \mathrm{meV}$.

The energy of optical phonon in this system is $31.5 \mathrm{meV}$, hence the last term in Eq. (1) is exponentially small for the temperature range below $100 \mathrm{~K}$. At the same time the acoustical phonon contribution is proportional to the temperature and dominates at low temperatures. An exciton scattering by the acoustical phonons is an elastic process. It means that the final electron state has the same module of the wave vector as the initial state. The maximal wave vector of an emitted or absorbed phonon is $q_{\max }=2 k$, where $k$ is the wave vector of the exciton. For the optically active exciton in the bulk material the exciton wave vector is $k \approx 10^{5} \mathrm{~cm}^{-1}$. Therefore, the only long wave phonons can participate in the exciton-phonon scattering. For the type-I QW one can show [4] that the maximal wave vector of the interacting phonon is $k \approx \pi / L_{\mathrm{QW}}=10^{6} \mathrm{~cm}^{-1}$. So the situation is changed. At the region outside the $\mathrm{QW}$ the hole wave vector is large enough (in parabolic approximation $k \approx \sqrt{2 m^{*} \varepsilon_{\mathrm{VBO}}} / \hbar \approx 4 \times 10^{7} \mathrm{~cm}^{-1}$ ), and the number of the phonons which can interact with the hole increases strongly for such state in comparison with the type-I QW and bulk materials.

A phonon broadening of the hole state can be estimated in the relaxation time approximation. In this approximation $\Gamma_{\mathrm{AC}}=\hbar / \tau$, where $\Gamma_{\mathrm{AC}}$ is an energy 


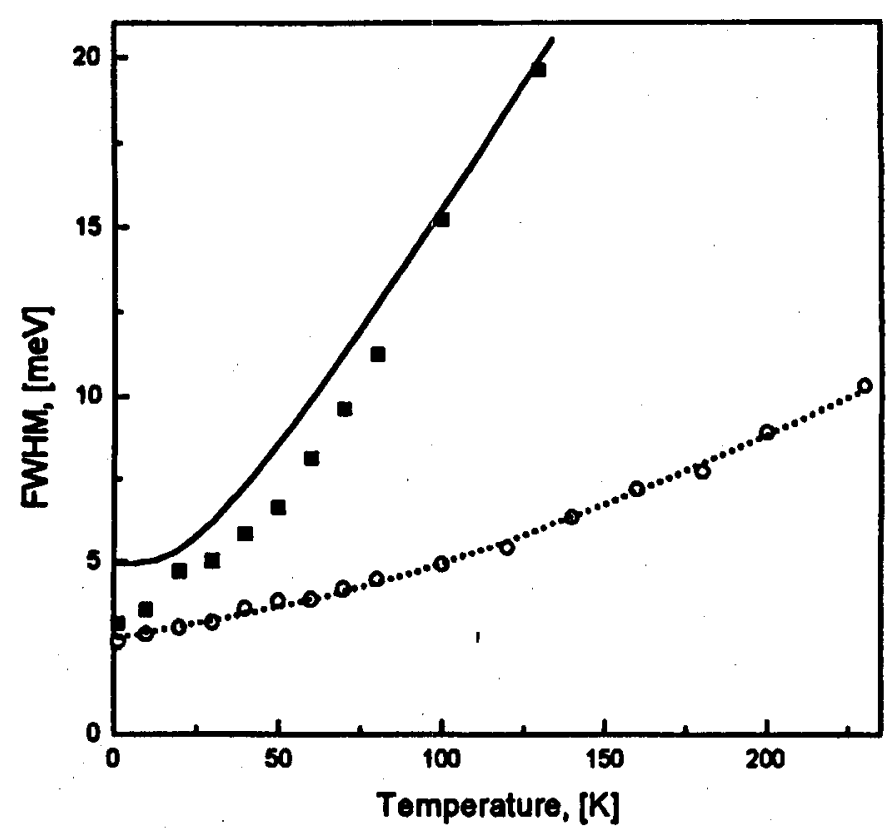

Fig. 3. A full width at a half maximum of the exciton resonance as a function of the temperature for the type-I $\mathrm{ZnSe} / \mathrm{BeZnSe} \mathrm{QW}$ (experiment - open circles, calculation — dotted line) and for the type-II $\mathrm{ZnSe} / \mathrm{BeTe} \mathrm{QW}$ (experiment - squares, calculation - solid line).

level broadening, $\tau$ is a relaxation time. We take into account the fact that outside the QW the hole has a kinetic energy which is much larger than the exciton binding energy and it reduces the problem to the scattering problem of a free particle. Using a deformation potential approximation the damping can be expressed as [5]

$$
\Gamma=\frac{\Omega_{0} m^{*} \varepsilon_{\mathrm{DF}}^{2}}{8 \hbar M v_{0} k^{3}} \int_{0}^{2 k}\left[1+2 /\left(\exp \left(\frac{\hbar v_{0} q}{k_{\mathrm{B}} T}\right)-1\right)\right] q^{4} \mathrm{~d} q,
$$

where $\Omega_{0}$ and $M$ is a volume and mass of a unit cell, respectively, $v_{0}$ is a longitudinal sound velocity, $\varepsilon_{\mathrm{DF}}$ is a deform-potential constant, $m^{*}$ and $k$ is effective mass and wave vector of the particle, respectively, $k_{\mathrm{B}}$ is the Boltzman constant, $T$ is a temperature.

The $\Gamma$ value is plotted in Fig. 3 (solid line). The following parameters were used $v_{0}=4 \times 10^{5} \mathrm{~cm} / \mathrm{s}, \varepsilon_{\mathrm{DF}}=5 \mathrm{eV}$. The values $m^{*}=1.5 m_{0}$ and $k=$ $1.35 \times 10^{7} \mathrm{~cm}^{-1}$ were found from the comparison with experiment. The calculated curve is very similar to the experimental one. At the high temperature limit Eq. (2) transforms into the second term in Eq. (1) with effective interaction constant $\gamma_{\mathrm{AC}}^{\mathrm{eff}}=\left(\Omega_{0} m^{*} \varepsilon_{\mathrm{DF}}^{2} k_{\mathrm{B}} / \hbar^{2} M v_{0}^{2}\right) k$. This quantity increases as $\sqrt{\varepsilon_{\mathrm{VBO}}}$ by increasing the negative band offset value $\varepsilon_{\mathrm{VBO}}$. At the low temperature limit $\gamma_{\mathrm{AC}} \propto \varepsilon_{\mathrm{VBO}}$ depends weakly on temperature. So, the above-barrier states have a large phonon contribution to the damping even at zero temperature. We note that the piezoelectric potential is of minor importance for the considered situation 
as $\gamma_{\mathrm{AC}}^{\text {piez }} \propto 1 / k \propto 1 / \sqrt{\varepsilon_{\mathrm{VBO}}}$. Hence, the efficiency of the exciton-acoustic phonon scattering via piezoelectric potential decreases for states with a larger $k$-value.

In conclusion, we have studied the optical spectra in the type-II $\mathrm{ZnSe} / \mathrm{BeTe}$ QW and observed strong exciton transitions involving the above-barrier quasi-localized hole states. We have found the drastic temperature increase in the exciton line width at low temperature range $(T<100 \mathrm{~K})$. The model is suggested to explain these results.

This work is supported in part by the Deutsche Forschungsgemeinschaft (SFB410), Volkswagen Foundation, INTAS grant No. 93-3657 Ext and by the Russian Foundation for Basic Research grant No. 95-02-04061a.

\section{References}

[1] A. Waag, F. Fisher, K. Schüll, T. Baron, H.-J. Lugauer, Th. Litz, U. Zehnder, W. Ossad, T. Gerhard, M. Keim, G. Reuscher, G. Landwehr, Appl. Phys. Lett. 70, 277 (1997).

[2] H. Luo, N. Dai, F.C. Zhang, N. Samarth, M. Dobrowolska, J.K. Furdyna, C. Parks, A.K. Ramdas, Phys. Rev. Lett. 70, 1307 (1993); N. Dai, L.R. Ram-Mohan, H. Luo, G.L. Yang, F.C. Zhang, M. Dobrowolska, J.K. Furdyna, Phys. Rev. B 50, 18153 (1994).

[3] E.L. Ivchenko, P.S. Kop'ev, V.P. Kochereshko, et.al., Sov. Phys. Semicond. 22, 784 (1988); E.L. Ivchenko, G.E. Pikus, Superlattices and other Heterostructures, in Springer Series in Solid-State Sciences, Vol. 110, Springer-Verlag, Berlin 1995.

[4] T. Takagahara, Phys. Rev. B 32, 7013 (1985).

[5] A.I. Anselm, Iu.A. Firsov, Sov. Phys. JETP 1, 139 (1955). 